\title{
Différentes définitions techniques de la forme des galets
}

\section{Different technical definitions of pebble shape}

\author{
PAR R. DURAND \\ INGÉNIEUR AUX ETAILISSEMENTS NEYRPIC
}

English synopsis p. 217

Nous avons été amenés, au cours d'études sur l'entraînement des matériaux par l'eau, à considérer l'influence de la forme de ces matériaux sur les lois qui régissent leur mouvement dans les fluides.

Dans cette première communication, nous avons simplement voulu faire l'examen des divers coefficients de forme actuellement définis afin de pouvoir choisir ensuite en connaissance de cause les mieux adaptés à chaque problème envisagé.

L'étude de la forme des galets inléresse l'hydraulicien à divers titres : vitesses d'entrainement, vitesse de chute, stabilité des plages et des digues en enrochements à la mer, écoulements en milieux poreux.

Elle intéresse également d'autres catégories de chercheurs que nous avons consultés :

- De façon générale, toute personne ayant à effectuer des analyses mécaniques et qui se préoccupe de la validité et de la signification de ses mesures.

- Les géologues qui examinent l'usure des galets pour en déduire les modes de transport successifs au cours des temps.

- Les techniciens des bétons qui se préoccupent de l'adhérence des liants aux agrégats, de la coulabilité et de la compacité des bétons.

- Les spécialistes de mécanique des sols qui étudient la porosité, la perméabilité et la cohésion des sols.

Lorsqu'il s'agit de définir la forme d'un galet ou d'un grain naturel quelconque, if vient naturellement à l'esprit de le comparer à une sphère ou à un ellipsoïde. Ceux-ci sont en effet, parmi les diverses formes idéales simples, les seuls qui ne comportent pas d'angles vifs et qui tiennent compte par conséquent du phénomène naturel de l'usure. Par suite, les coefficients de forme s'efforcent en général d'établir une comparaison avec une sphère ou un ellipsoïde de référence.

Nous distinguerons d'abord, parmi l'ensemble de ces coefficients de forme, ceux qui caractérisent le grain isolé en lụi-même, indépendamment de sa situation dans un ensemble ou dans un écoulement. Nous appellerons de tels coefficients, coefficienls « intrinsèques » de forme. Il s'agit de coefficients véritables, c'est-àdire sans dimensions. On peut ensuite pour chaque technique particulière être amené à définir des coefficients spéciaux. Le grain ne sera plus considéré isolément mais en relation avec d'aulres grains ou avec un fluide en mouvement. Il s'agira par exemple d'un coefficient caractérisant la position d'un galet sur le terrain pour le géologue ou dụ coefficient de traînée caractérisant la résistance aux déplacements du grain dans un fluide pour l'hydraulicien ou l'aérodynamicien. Bien souvent ces coefficients ne seront pas sans dimensions. Ainsi, la perméabilité est une vitesse fictive. Nous nous attacherons plus spécialement à l'étude des coefficients spéciaux définis en hydraulique.

De même nous nous intéresserons de préférence aux graviers et galets, c'est-à-dire aux grains supérieurs à $2 \mathrm{~mm}$. 


\section{CHAPITRE I}

\section{ETUDE DES COEFFICIENTS “INTRINSEQUES" DE FORME}

Nous distinguerons encore, parmi les coefficients « intrinséques », trois classes suivant que les grandeurs intervenant dans ces coeficients auront été mesurées suivant des droites, dans un plan ou dans l'espace.

Les grandeurs mesurables suivant une direction, grandeurs dites du premier ordre, sont les dimensions droites mesurées au pied à coulisse, au palmer ou au microscope avec micrometre.

Les mesures dans un plan, ou du second ordre, nécessitent la projection du contour apparent du grain suivant une direction choisie. Cette projection peut être réalisée au jugé ou à la chambre claire. On pourra alors effectuer les mesures suivantes : rayons de courbure, angles si le contour apparent affecte une allure polygonale, périmetre et surface intérieure.

Les mesures dites dans l'espace ou du troisième ordre sont des mesures de poids ou de volume effectuées à la balance. Elles peuvent donc être très précises si les grains sont assez gros.

Les coefficients correspondants seront dits du premier, du second ou du troisieme ordre. Ils représentent le grain de façon d'autant plus complète et complexe à la fois qu'ils seront d'un ordre plus élevé.

\section{$1^{\circ}$ Coefficients du premier ordre}

Nous admettrons que les trois dimensions principales du grain sont toutes définies comme étant les plus grandes longueurs mesurables entre deux plans, parallèles entre eux, et pouvant être parallèles eux-mêmes à une ou plusieurs directions données.

Nous reviendrons plus loin sur la validité de ces définitions.

Soit L, la longueur, qui est la plus grande dimension mesurable entre deux plans parallèles.

Soit L', la largeur, qui est la dimension normale à la direction de $\mathrm{L}$.

Soit L', l'épaisseur, qui est la dimension normale à la fois aux directions de $\mathrm{L}$ et de $\mathrm{L}^{\prime}$.

Les trois segments correspondants ne sont pas nécessairement concourants. La longueur peut être mesurée facilement et avec une précision suffisante. Par contre, il n'est pas toujours aisé de déterminer pratiquement la direction suivant laquelle doivent être mesurés $\mathrm{L}^{\prime}$ ou $\mathrm{L}^{\prime \prime}$ si le grain est trop petit pour pouvoir être tenu commodément à la main. Par observation au microscope, on ne peut en effet étudier que des positions d'équilibre stables du grain sur un plan et ces directions ne coincident pas nécessairement avec celles de $L^{\prime}$ et de $L^{\prime \prime}$.

On appelle diametre moyen $\mathrm{D}_{m}$ le diametre de la sphère ayant même volume que l'ellipsoïde ayant pour axes $\mathrm{L}, \mathrm{I}^{\prime}$ et $\mathrm{L}^{\prime \prime}$.

$$
\mathrm{D}_{m}=\sqrt[3]{\mathrm{L} X \mathrm{I}^{\prime} \times \mathrm{I}^{\prime \prime}}
$$

\section{Coefficient d'aplatissement ou flatness ratio.}

Wentworth, Wadell et Cailleux définissent l'aplatissement ainsi :

$$
\frac{\mathrm{L}+\mathrm{L}^{\prime}}{2 \mathrm{~L}^{\prime \prime}} \geqslant 1
$$

en fait, il varie de 1,05 à 10 d'après Carluevx.

On peut utiliser (Mc Nowv) un coefficient d'aplatissement correspondant à une moyenne géométrique de la forme :

$$
\frac{\mathrm{L}^{\prime \prime}}{\sqrt{\mathrm{I} X \mathrm{~L}^{\prime}}} \leqslant 1
$$

\section{Coefficient de dissymétrie.}

Si $\mathrm{C}$ est le point où le plan contenant $\mathrm{L}^{\prime \prime}$ coupe $\mathrm{L}$ et si A est l'extrémité de L la plus éloignée de $C$, Callleux appelle dissymétrie le rapport :

$$
0,5<\frac{\mathrm{AC}}{\mathrm{L}}<1
$$

Pratiquement, il varie entre 0,5 et 0,85 .

La notion d'aplatissement peut varier suivant que l'on fait intervenir deux ou trois dimensions (étude en plan ou en volume) et suivant que l'on tient ou non compte de la dissymétrie du grain.

CaIlleux utilise ainsi un coefficient d'aplatissement en section $\frac{\mathrm{L}}{\mathrm{L}_{\mathrm{s}}}$. L'épaisseur $\mathrm{L}_{\mathrm{s}}^{\prime}$ est alors la plus grande épaisseur normale à L et peut différer de $L^{\prime \prime}$. 


\section{Indice granulaire.}

La norme autrichienne Onorm B 3102 définit l'indice granulaire (Kornindex) comme étant le rapport de la longueur L à l'épaisseur E mesurée dans le plan médiateur de $\mathrm{L}$. Cette épaisseur E n'est donc égale à $L^{\prime \prime}$ que si la dissymétrie est de 0,5 .

\section{Modules d'allongement et d'aplatissement.}

K. WaLz mesure $L$ et $L^{\prime}$. Il mesure en fait l'épaisseur $e$ au milieu de $\mathrm{L}$ et normalement à $\mathrm{L}$ et à $L^{\prime}$, mais pour simplifier nous continuerons à désigner l'épaisseur par $L^{\prime \prime}$. Il considère les rapports $\frac{L^{\prime}}{L^{\prime \prime}}$ et $\frac{L}{L^{\prime \prime}}$. En portant en abscisses $\mathrm{L}^{\prime \prime}$ et en ordonnées $\frac{\mathrm{L}^{\prime}}{\mathrm{L}^{\prime \prime}}$, on obtient le graphique de la figure 1. Les limites sont constituées par les droites SA et SD. Le point S correspond $\grave{a}$ la sphère et les directions $A$ et $D$ respectivement aux aiguilles et aux disques. On peut distinguer quatre zones I, II, III, IV, qui caractirisent la tendance à la sphéricité et deux autres zones séparées par la droite SM qui distinguent les allongés et les aplatis.

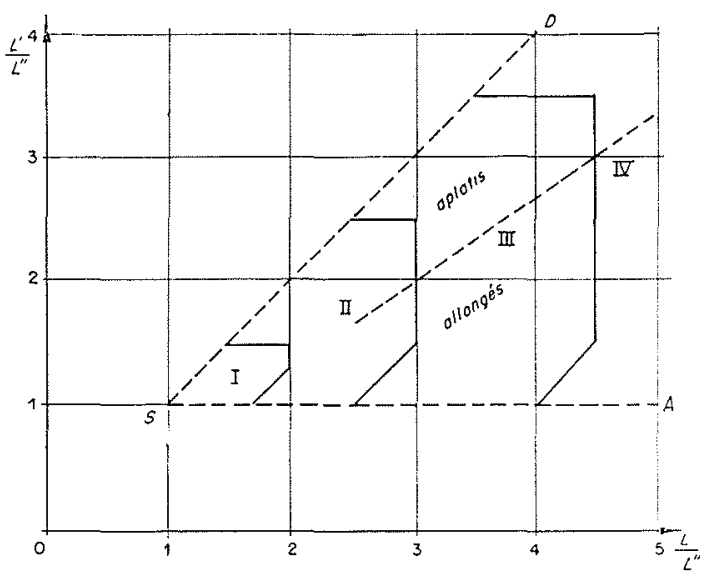

Fig. 1. - Diagrame de K. WaLz

M. A. H. D. Markwick définit le module d'aplatissement par $\frac{L^{\prime \prime}}{L^{\prime}}$ et le module d'allongement par $\quad L^{\prime}$. Il considere comme aplatis les grains pour lesquels $\frac{\mathrm{L}^{\prime \prime}}{\mathrm{L}^{\prime}}<0,6$ et comme allongés ceux pour lesquels $\frac{\mathrm{L}}{\mathrm{L}^{\prime}}>1,8$.

Il nous semble préférable de prendre comme terme de comparaison commun la largeur et d'adopter pour les modules les définitions de H. HEYwood, e'est-à-dire module d'aplatissement égal à $\frac{L^{\prime}}{L^{\prime \prime}}$ et module d'allongement égal à $\frac{L}{L^{\prime}}$. En adoptant ces définitions, les deux modules sont supérieurs ou igaux à l'unité.

Si l'on porte en abscisses $n=\frac{\mathrm{L}}{\mathrm{L}^{\prime \prime}}$ et en ordonnées $m=\frac{\mathrm{L}}{\mathrm{L}^{\prime}}$ à la manière de $\mathrm{K}$. Walz, le point $S$ de la figure 2 représente la sphère, et

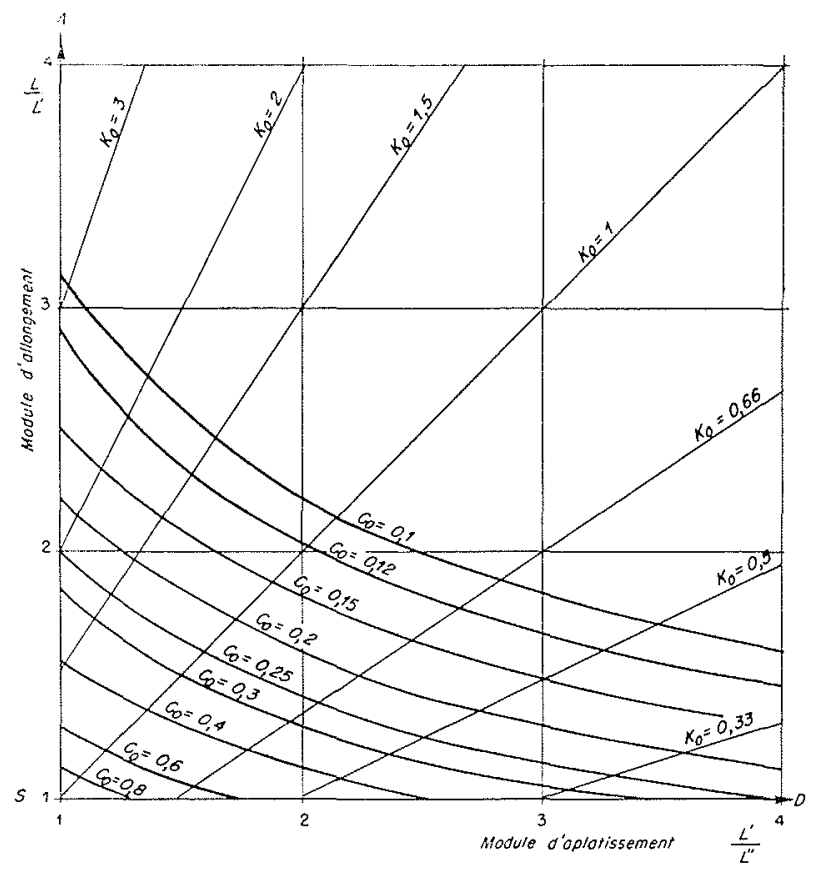

FIG, 2.

les directions $\mathrm{A}$ et $\mathrm{I}$ l'aiguille et le disque. La droite $S$, de pente unité, passant par $S$ et pour laquelle $\mathrm{K}_{0}=\frac{\mathrm{L}_{\mathrm{L}}{ }^{\prime \prime}}{\mathrm{L}^{\prime 2}}=1$, sépare le quadrant en deux régions :

- la région supérieure correspond à $\mathrm{K}_{0}>1$ et aux grains allongés,

- la région inféricure correspond à $K_{0}<1$ et aux grains aplatis.

A une valeur donnée de $K_{0}$ correspond une droite passant par l'origine de pente $\mathbf{K}_{0}$. Le point correspondant à un grain dans le diagramme des modules définit ainsi son ellipsoide de référence.

En résumé, pour définir l'aplatissement avec un seul coefficient, on prendra :

- soit l'aplatissement en section de Carllevx ou l'indice granulaire qui ne nécessitent que deux mesures,

- soit l'aplatissement de Wadell ou celui de Mc Nown qui en nécessitent trois.

Si l'on veut distinguer entre l'aplatissement et 
l'allongement, on utilisera les modules d'Heywoon et le coefficient $\mathbf{K}_{0}$.

\section{$2^{\circ}$ Coefficients $\mathrm{du}$ second ordre}

Les mesures sont faites sur le contour apparent du grain projeté dans la direction normale $\dot{a} \mathrm{~L}$ et $\mathrm{L}^{\prime}$ (contour apparent principal). Souvent ce contour principal correspond à la position d'équilibre la plus stable du galet. Pour les grains fins, dont l'observation doit se faire au microscope, cette définition est d'ailleurs la seule possible.

\section{a) Coefficients d'arrondi.}

Ces coefficients font intervenir les rayons de courbure du contour apparent. On mesure généralement le plus petit rayon de courbure. On ne considère pas les petits détails correspondants à l'état de surface (grains constitutifs pour les quartzites ou petites cavités résultant du départ d'un éclat sur les silex). On rapporte ensuite le double de ce rayon à une dimension caractéristique du grain. On obtient, suivant la dimension de référence :

- Roundness de Cox \& WextworTH :

$$
\frac{2 r_{1}^{\prime}}{\mathrm{D}_{m}}
$$

-n.- Indice d'émonssé de Canlleux :

$$
\frac{2 r_{1}}{\mathrm{~L}}
$$

- Roundness de WADELL :

$$
\frac{2 r_{1}}{\mathrm{D}}
$$

avec $D$ plus grand cercle inscrit dans le contour apparent; D correspond souvent pour les galets roulés à la largeur.

Wadell \& CaIllevx mesurent également les rayons de courbure supérieure à $r_{1}$. WADELL, peut ainsi distinguer jusqu'à 7 rayons principaux sur un contour apparent. Il prend alors la moyenne pour $\mathrm{N}$ rayons de courbures et appelle plus précisément roụndness

$$
\mathrm{R}=\frac{2 \Sigma(r)}{\mathrm{D} \cdot \mathrm{N}}
$$

Tester définit par ailleurs un coefficient pour les grains à allure polygonale qui semble difficile à déterminer avec précision.

\section{b) Coefficients de circularité.}

Pour le contour apparent, on peut encore mesurer :

- L et L', la longueur et la largeur déjà définies,

- $s$, la surface du cercle de diamètre $L$,

-. p, le périmètre du contour apparent,

- $s_{0}$, la surface du contour apparent.

- $\mathrm{D}_{0}$, le diamètre du cercle de surface $s_{0}$,

- $p_{0}$, le périmètre du cercle de surface $s_{0}$.

Avec ces six grandeurs, les différents auteurs ont pu définir des coefficients dits de « circularity $\gg$ ou de «sphericity».

Coefficient de $\operatorname{Cox}: 4 n \frac{s_{0}}{p^{2}}$.

Coefficient de Tickell : $\frac{s_{0}}{s}$.

Coefficient de Heywoon et de Markwak :

$$
x=\frac{s_{0}}{\mathrm{~L} \mathrm{~L}^{\prime}}
$$

Coefficients de WADELL : $\frac{p_{0}}{p}$ et $\varphi=\frac{D_{0}}{L}$.

Pour des grains de quartz d'allure sphérique, WADELL mesure $\varphi$ suivant différents contours apparents, et la moyenne om lui semble peu différente de la « true sphericity $\psi \psi$ définie ellemême par :

$$
\psi=\frac{\mathrm{S}_{0}}{\mathrm{~S}}
$$

avec :

$\mathrm{S}_{0}$, surface latérale de la sphère de même volume, $\mathrm{S}$, surface réelle du grain.

$S$ n'est pas une grandeur pratiquement mesurable et il faut remarquer que $\varphi$ est un rapport de longueurs et $\psi$ un rapport de surfaces. La notion de 9 moyen nous semble done présenter peu d'intérèt.

\section{$3^{\circ}$ Coefficients du troisième ordre}

Tous ces coefficients sont basés sur la mesure du poids et par conséquent du volume du grain.

La norme AFNOR P-18 301 désigne par coefficient volumétrique $\mathrm{C}$ le rapport du volume $\mathrm{V} d u$ grain au volume de la sphère circonscrite, c'est$\grave{a}$-dire ayant pour diamètre la longueur $\mathrm{L}$; et par 
coefficient volumétrique moyen $\mathrm{C}_{m}$, le rapport du volume absolu total de $N$ grains à la somme des volumes des sphères ayant pour diamètre la longueur des grains.

$$
\mathrm{C}_{m}=\frac{\mathrm{vV}}{\mathrm{\Sigma} \frac{\pi \mathrm{L}}{6}}
$$

Heywood appelle « volume constant» $k$, le rapport du volume $V$ de la particule à $\left.D_{0}\right)^{3}$, volume du cube ayant pour arcete $D_{0}$.

$$
k=\frac{\mathrm{V}}{\mathrm{D}_{0}{ }^{3}}
$$

ANdrasen définit un coefficient égal à :

$$
\frac{1}{3 \sqrt{k}}=\frac{D_{0}}{3 \sqrt{V}}
$$

Makwick appelle « volume ratio»:

$$
\frac{\mathrm{V}}{\mathrm{LL} \mathrm{L}^{\prime \prime}}
$$

ct « prismoïdal ratio» :

$$
\frac{\mathrm{V}}{s_{0} \mathrm{~L}^{\prime \prime}}
$$

Soit $\mathrm{D}_{n}$ le diamètre nominal, c'est-à-dire le diametre de la sphère ayant même volume que le grain :

$$
\mathrm{D}_{n}=\sqrt[3]{\frac{6 \mathrm{~V}}{\pi}}
$$

Makkwick appelle « surface constante » $\sigma$ le rapport :

$$
\sigma=\frac{\mathrm{L}^{\prime}}{\mathrm{D}_{n}}
$$

Frret appelle par ailleurs volume relatif $K_{m}$ le volume moyen de $\mathrm{N}$ grains rapporté à la moyenne des volumes des sphères traversant juste les passoires ou les deux mailles ayant servi à isoler la catégorie de grains considérée. Ce coefficient ne peut s'appliquer qu'à des matériaux tamisés.

En ce qui concerne la mesure du coefficient volumétrique moyen pour des grains fins, si l'on opère sur un nombre de grains limité, la pesée est peu précise; si l'on opère sur un nombre important, la méthode devient fastidieuse étant donné le grand nombre de mesures de $\mathrm{L}$ au microscope à effectuer.

Pour des matériaux classés, la notion de voIume relatif est aisce à déterminer puisqu'il suffit de compter les grains.

\section{CHAPITRE II}

\section{EMPLOI DU DIAGRAMME DES MODULES}

Les dimensions principales du grain peuvent être définies de différentes façons.

Nous distinguerons les dimensions $L, L^{\prime}, L^{\prime \prime}$ qui correspondent au plus grand parallélépipède circonscrit, et les dimensions $l, l^{\prime}, l^{\prime \prime}$ qui correspondent au plus petit parallélépipède circonscrit.

Pour les ellipsoïdes et les galets roulés en général, l'écart entre les deux définitions est nul ou négligeable. Il devient important pour les blocs anguleux et pour le cube ou les parallélépipèdes.

Un autre point de vue est celui de l'équivalence entre les dimensions définies entre plans parallèles et les dimensions définies par analyse mécanique (tamis à maille carréc ou rectangulaire, fentes, passoires).

L'écart est également nul pour la sphère et l'ellipsoïde, faible en général pour les cailloux roulés et grand pour les concassés et les polyèdres.

Après avoir fait ces remarques, nous allons maintenant préciser la notion de forme. WaDFL. estime que, pour avoir une bonne image du galet, il faut examiner à la fois "sphericity" ou "circularity" et "roundness".

Markwick distingue également entre «shape » et « form ». Dans le même esprit, nous parlerons d'allure et d'arrondi. L'allure implique l'idce d'allongement et d'aplatissement plus ou moins grand. L'arrondi permet de distinguer les galets roulés des blocs anguleux concassés.

Nous allons voir comment, sur le diagramme des modules que nous avons choisi, on peut caractériser l'allure et l'arrondi.

L'allure dụ galet sera définie par les valeurs des modules d'allongement et d'aplatissement $n$ et $m$.

La pente de la droite joignant le point de coordonnées $n$ et $m$ à l'origine est :

$$
\mathrm{K}_{0}=\frac{\mathrm{LL}^{\prime \prime}}{\mathrm{L}^{\prime 2}}
$$


Le coefficient $K_{0}$ devient, si l'on multiplic numérateur el dénominateur par $\frac{\pi}{6} \mathrm{~L}$, le rapport du volume de l'ellipsö̈de de référence ayant pour axes $L, L^{\prime}, L^{\prime \prime}$, au volume de la sphère ayant pour diamètre $L^{\prime}$, c'est-à-dire pratiquemen! le volume de la sphère passant juste à la passoire qui définit le grain.

Soient alors :

$K_{0}=$ volume relatif de l'ellipsoïde de référence,

$K=$ volume relatif vrai d'un grain isolé,

$\mathrm{K}_{m}=$ volume relatif vrai moyen d'un ensemble de grains déjà définis,

$\mathrm{C}_{0}=$ coefficient volumétrique de l'ellipsoïde de référence,

$\mathrm{C}=$ coefficient volumétrique vrai d'un grain isolé déjà défini,

$\mathrm{C}_{m}=$ coefficient volumètrique moyen d'un ensemble.

Comme $C_{0}=\frac{L L^{\prime} L^{\prime \prime}}{L^{3}}$, on en tire :

$$
m=\frac{l}{\sqrt{\mathrm{C}_{0} n}}
$$

Remarquons que $C_{0}$ varie davantage avec l'allongement qu'avec l'aplatissement.

On peut graduer le diagramme en courbes d'égal $\mathrm{K}_{0}$ et d'égal $\mathrm{C}_{0}$. Si l'on peut déterminer la valeur de $\mathrm{K}$ ou de $\mathrm{C}$, le rapport $\frac{\mathrm{K}}{\mathrm{K}_{0}}$ ou $\frac{\mathrm{C}}{\mathrm{C}_{0}}$ pourra caractériser l'arrondi du grain.

Remarquons que $\frac{\mathrm{K}}{\mathrm{K}_{0}}$ et $\frac{\mathrm{C}}{\mathrm{C}_{0}}$ sont l'un et l'autre le "volume ratio» de Mankwick :

$$
\mathrm{V}_{0}=\frac{\mathrm{V}}{\mathrm{L} \mathrm{L}^{\prime} \mathrm{L}^{\prime \prime}}
$$

On peut par suite considérer indifféremment l'un ou l'autre.

Comme l'a déjà fait WaLz, on peut distinguer dans le diagramme différentes zones correspondant à des familles de galets. Par exemple, nous avons pu constater que les galets du Rhône à Donzère se placent dans une bande axée sur la droite $K_{0}=1$.

\section{CHAPITRE III}

\section{APPLICATIONS AUX DIFFERENTES TECHNIQUES}

Pour l'étude expérimentale de l'influence des facteurs de forme sur un phénomène, on peut choisir des galets presque identiques et ne différant entre eux que par un seul de ces coefficients. Les galets seront alors mesurés par les méthodes déjà indiquées.

Pour l'application de ces lois à un échantillon naturel complexe, qui nécessite des mesures statistiques sur un grand nombre de grains, il faut faire appel à des procédés de classement mécaniques.

On ne peut malheureusement pas mesurer la longueur par des procédés mécaniques simples. L'emploi de tamis à mailles carrées ou de passoires à orifices circulaires permet de classer l'échantillon en plusieurs catégories dont chacune est constituée de grains ayant sensiblement la même largeur. En analysant ces catégories grâce à un système de fentes de largeurs différentes permettant la mesure de l'épaisseur, on peut définir des modules d'aplatissement moyens.

Le diamètre $\mathbf{D}_{0}$ défini par tamisage à la passoire differe souvent de la largeur que nous avions primitivement définic. Hrywoon signale que le diamètre statistique de MarTis se rapprocherait davantage de $D_{0}$ (que $L^{\prime}$. Martin considère la largeur suivant la droite passant par le centre de gravité de l'aire projetée.

Enfin, la valeur du vide de maille carrée laissant passer le même grain que la passoire $D_{0}$ est une fonction à préciser du module d'aplatissement.

\section{GÉOLOGIE}

Le géologue cherche à déduire de la forme du galet et de sa position par quel processus il a été amené sur le terrain. C'est grâce au phénomène d'usure qu'il peut déterminer si le grain est d'origine marine, fluviatile, éolienne, etc... On s'efforcera d'établir une comparaison avec une sphère de référence ou un ellipsoïde. L'idéal serait de comparer la surface réelle du grain à celle de la sphère de même volume. La mesure de la surface réelle étant impossible, on doit se 
contenter de l'étude dans le contour apparent principal, d'où les différents coefficionts de circularité. Une autre voie de recherches est orientée vers la mesure des rayons de courbure.

On pourrait peut-être utiliser des coefficients volumétriques basés sur une mesure de poids Lels que $\frac{\mathrm{K}}{\mathrm{K}_{0}}$ ou $\frac{\mathrm{C}}{\mathrm{C}_{0}}$.

\section{TRAVAUX PUBLICS}

Pour l'étude des bétons, on se préoccupe de la rondeur pour l'adhérence du liant à l'agrégat, et de l'allure pour la coulabilité. Pour les chaussées empierrées, on s'intéresse principalement aux grains concassés et peu aplatis.

On considère toujours de grandes quantités de matériaux, de sorte que l'on est obligé, pour considérer des valeurs moyennes, de faire appel systématiquement à l'analyse mécanique.

\section{MÉCANIQUE DES FLUIDES}

L'hydraulicien considère toujours le grain en mouvement relatif par rapport à un fluide. On peut donc penser que les coefficients les mieux adaptés à ce sujet seront les coefficients de rolume du troisième ordre.

Nous distinguerons les cas suivants :

\section{a) Ecoulements en milienx poreux :}

Le coefficient de perméabilité $K$ de la formule de Darcy est une vitesse fictive qui dépend du fluide et de la forme des grains. L'indice des vides peut également constituer un facteur de forme.

b) Tenue dine plage, de massifs d'cnrochements naturels et de blocs artificiels at la mer:

La stabilité d'une plage ou d'une digue à la houle est fonction des caractéristiques locales de la houle et de son incidence, de l'angle du talus et du poids et de la forme des constituants. On a pu constater par des essais que l'on pouvait nettement améliorer la stabilité en jouant sur la forme denrochements artificiels.

\section{c) Vitesses dentrainement:}

On peut distinguer plusieurs vitesses d'entraînement :

1. Vitesse d'entrainement sur fond lisse;
2. Vitesse d'entrainement sur fond rugueux constitué par des matériaux analogues (H.IULSTROM);

3. Vitesse d'entrainement pour des massifs en pierres lancées (Théorie d'IsBucn et Branchet).

Pour chacune de ces vitesses il faut faire intervenir un facteur de forme différent. En effet, l'entrainement sur fond lisse est un phénomène de glissement ou de roulement. L'entrainement sur fond rugueux comporte un pivotement.

\section{d) Vitesses de chute:}

Lorsqu'un grain isolé de maitre couple $S$ a atteint sa vitesse limite $W$ dans un fluide indéfini, de masse spécifique o, il y a équilibre entre son poids apparent $P_{a}$ et la trainée :

$$
\mathrm{P}_{a}=\mathrm{C}_{x} \circ \mathrm{S} \frac{\mathrm{W}^{2}}{2}
$$

Le coefficient $\mathrm{C}_{r}$ ainsi défini, appelé coefficient de trainée, varie avec le nombre de REyNoLDs $\frac{W D}{\nu}(D$, diametre du grain et $y$, viscosité cinématique du fluide). Il est sans dimensions.

Pour de faibles nombres de Rxrowos el pour des sphères, on a $\mathrm{C}_{x^{\prime}}=\frac{24}{\mathrm{R}}$ qui correspond à la loi de Stores. Pour des nombres supérieurs de Rervolds compris entre $10^{*}$ et $10^{5}, \mathrm{C}_{j}$ reste pratiquement constant. Me Nown a étudié le coefficient de traince des ellipsoïdes et de corps artificiels de forme géométrique aux faibles nombres de Rexnouns. Il est amené à définir le coefficient $K$, rapport de la trainéc réelle du corps considéré à la traînće fictive de la sphère ayant même volume et mème vitesse de chute. K est également le rapport de la vitesse de chute de la sphère de même volume à la vitesse de chute du corps.

On appelle diametre équivalent $\mathrm{D}_{\text {c }}$ d'un grain le diamètre de la sphère ayant mème vitesse limite de chute W.

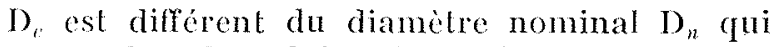
est, rappelons-le, celui de la sphère de mème volume. $\frac{D_{0}}{D_{n}}$ peut constituer un facteur de forme.

Nous avons nous-mèmes été amenés à étudier les vitesses de chute et le coefficient de trainée de galets roulés du Rhòne en vue de leur refoulement en conduite. Nous avons pu constater que le coefficient volumétrique $\mathrm{C}$ était suffisant pour déterminer la valeur de $\mathrm{C}_{x}$. Ceci implique qu'une certaine relation existait entre les coefficients de forme de ces galets. On peut done, dans ce cas, tracer des courbes d'égal $C_{x}$ sur le diagramme des 
modules. Nous envisageons de eompléter cette étude pour d'autres types de galets et pour des sables dans le cadre d'une étude entreprise pour les Ponts et Chaussées de la Loire-Inférieure.

\section{CONCLUSION}

Dans cette étude, nous avons essayé de classer les coefficients intrinsèques de forme en trois classes suivant la nature des mesures à effectuer. On peut distinguer l'allure du galet et son arrondi. L'allure peut être mesurée grâce à des rapports de longueurs appelés modules d'aplatissement et d'allongement.

La position du point représentalif du galet dans le diagramme des modules représente directement lallure du grain, et le classe dans une famille. Le diagramme associe au grain un ellipsoïde de comparaison de mêmes axes.

L'arrondi pourra être caractérisé, grâce à une mesure de volume, par l'écart entre un coefficient volumétrique du grain et le coefficient volumétrique de l'ellipsoïde.

Nous espérons que ce travail de documentation pourra donner quelques idées directrices pour l'étude physique des propriétés des sables et des galets en hydraulique.

\section{NO'TATIONS}

Pour donner plus de clarté à l'exposé, nous avons désigné arbitrairement par la même lettre les grandeurs qui étaient désignées difréremment par plusieurs auteurs.

$\mathrm{L}=$ Iongueur maxima.

$\mathrm{L}^{\prime}=$ largeur maxima perpendiculaire à $\mathrm{L}$.

$\mathrm{L}^{\prime \prime}=$ épaisseur maxima perpendiculaire à $\mathrm{L}$ cet $\mathrm{L}^{\prime}$.

$\mathrm{L}^{\prime \prime}{ }_{8}=$ épaisseur maxima perpendiculaire ì $\mathrm{L}$.

$\mathrm{E}=$ plus petite épaisseur dans le plan médiateur de L.

$e=$ épaisseur au milieu de $\mathrm{L}$ perpendiculairement à $\mathrm{L}$ et à $\mathrm{L}^{\prime}$.

$r_{1}=$ plus petit rayon de courbure du contour apparent principal.

$\mathrm{D}=$ plus grand cercle inscrit dans le contour apparent principal.

$\mathrm{S}=$ surface du cercle de diamètre $\mathrm{L}$.

$p=$ périmètre du contour apparent principal.

$S_{0}=$ surface du contour apparent principal.

$\mathrm{D}_{0}=$ diamètre du cercle de surface $\mathrm{S}_{0}$.

$p_{0}=$ périmètre du cercle de surface $S_{0}$.

$m=$ module d'allongement.

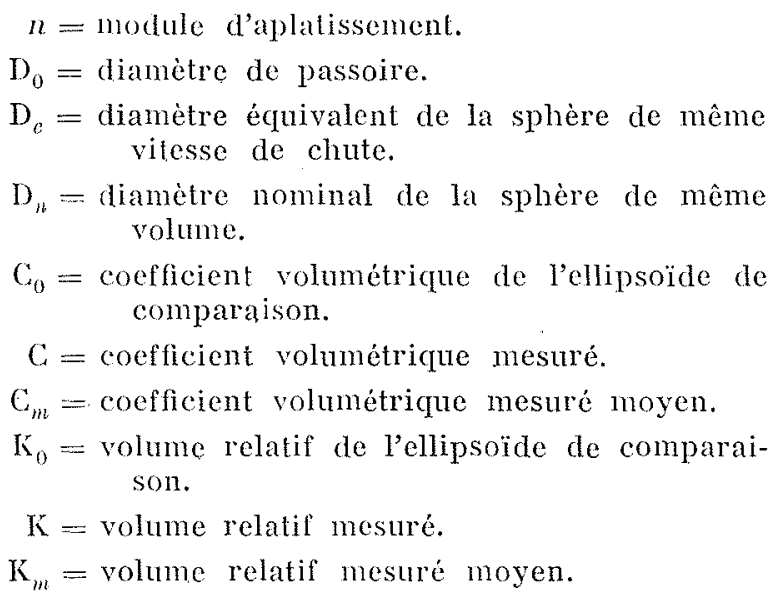

\section{BIBLIOGRAPHIE}

Nous présenterons à part les références bibliographiques des géologues et des spéeinlistes de bétons et de mécanique des sols, qui présentent peu d'auteurs communs et semblent souvent s'ignorer.

\section{GÉOLOGIE.}

Andinson. - The rate of wear of Sand Grains. Journal of Geology, Vol, 34 (1926), pp. 144-158.

Bourcart. - Essai d'une elassification des matériaux meubles. Extrait du Bulletin de la Société Géologique de France, 5" série, T. XI, annéc 1941.

Calltax (A.). - Indice d'émoussé. Définition et première application. Extrait du C.R.S. de la Société Géologique de France, séance du 10 nov. 1947, p. 251.

Cambeux (A.) el Boumber (Geneviève). - Indice d'èmoussé des galets de quartz. Extrat du C.R.S. de la Sociélé Géologique de France, séance du 24 janvier 1949,1 , 35.

Canlleux (A.) el Boullat (Geneviève). - Indice d'émoussé du silex. Extrait du C.R.S. de la Société Géologique de France, séance du 10 mai 1948, p. 1.947 .

Carluevx (A.). -.. La disposition individuelle des galets dans les formations détritiques. Revue de Géographie physique et de Géologie dynamique, 1938, Vol. XI, fasc. 3, pp. 171-198.

Callevex (A.). - Distinction des galets marins et fluviatiles. Extrait du Bulletin de la Société Géologique de France, $5^{\circ}$ série, T. XV, année 1945 .

Carthy (Mac). - The rounding of beach sands. American Journal Sci., Vol. CCXV (1933), pp. 205-224.

Cox (E. P.). - A methode of assigning numerical and percentage values to the degree of roundness of sand grains. Journal of Paleon, Vol. I (1927), pp. 179-183.

Lamar (T. E.). - Geology and economic resources of the St. Peter Sandstone of Illinois. Ill. Geol. Sur. Bulletin, 53,1927 , pp. $148-151$.

Macke. - On the laws that govern the rounding of particles of sand. Trans. Edin. Geolog. Society, Vol. VII (1893-1898), p. 299-311.

Marshart (P.). - The wearing of beach gravels. Trans. New Zealand Inst., Vol. LVIII (1927), pp. 507-532.

SiNGH (Harbans). - "Roundness" and "Sphericity" as used in analysis of sand particles. The central board of irrigation journal, March 1950, p. 163. 
Thester (A.). - The measurement of the shapes of rock particles. Journal Sedim. Petrology, Vol. I, 1931, pp. 3-11.

Traken, ( $F$. ( $x$.) - - The examination of fragmental rocks. Stanlord University press (1931).

WaDeli (H.). - Volume, shape and roundness of rock particles. Jonmal of Geology, July-August 1932, Vol. XL, n" $1^{\prime \prime}, \mathrm{pp}, 4+43-451$.

Waners (H.). - Sphericity and roundness of rock particles. Journal of Geology, April-May 1933, Vol. XLI, n" 3, pp. 310-331.

Wabele (H.). -- Volume, shape and loundness of quart $x$ particles, Journal of Geology, April-May 1935, Vol. XLIII, $\mathrm{n}^{* 3}$, pp. 250-280.

TVextworth. - - The shapes of pebbles. United States Geological Survel, Bulletin 730 C, 1922, pp. 91-114.

Westwortr. -.. The shapes of beach pebbles. United States Geological Survey. Professional paper, $131 \mathrm{C}$, pp. $75-83$.

\section{BÉTON ET MÉCANIQUE DES SOLS.}

ANon. - Proposed standard specification for concrete aggregates and for mixing water. Annales de l'Institut Technique du Batiment et des Tranalux Publics, 1936, I (2), p. 73 .

Andreasen (A. M. H.). - Zur keuntnis des Mahlgutes lollodehem Beihefte, 1928-1927, p. 349.

Chements. - Particle shape and surface characteristies of aggregates. Chemistry and industry, March 13, 1937, p. 248 .

Dunizz. - Matériaux de Construction. 1950.

Favny. - Le Béton. Dunod, 1942.

Fíner (R.). - La grosseur des grains de matière pulvérulente. Assoc. Inter. pour l'essai des matériant. Zurich, 1931, 11, Group. D.

Foñer (R.). - Sur la forme et l'état de surface des éléments inertes des bétons. Annales de l'Inst. Techn. dut Batiment et des Trabaux Publies, mars-avril 1937, p. 52 .

Fúnge (R.). - Nouvelles recherches sur la forme des éléments inertes des bétons. Annales de l'Inst. Techn, du Bä́iment et des Travatux Publics, mai-juin 1938.

Fujn (M.). - Effects of forms of particles upon the technical properties of argregates. Research office of public Worlis, Tokio, 1935.

Herwood (H.). - Numerical definitions of particles size and shape. Joumal of the Society of Chemical Industry, Vol. LVI; Chemistry and Industry, Vol. XV, 1937, Feb. $13,1937$.

Herwoon (H.). - The calculation of the specific surface of a powder. Inst. Mech. Eng. Proce, 1933, 125, pp. 383459.

Markwick (A. H. D.). - The shape of Crushed stories and gravel and its measurement. Journal of the Society of Chemical Industry, Vol. LVI; Chemistry and Industry, Vol. XV, 1937, Feb. 27, 1937.

Martix (G.). - Research on the Theory of fine grinding lrans. Ceramic Society, 1923, 4, 23, p. 61.

Orzex (R.). - Wertziffern für die Kornform ron Splitten und Grïsen. Zement, 1929, Vol. 18, p. 300.

Rothrugrs (G.). - Bewertung der Versehiedenartigen. Kornform von Steinschlag und Splitt. Zement, 1931, Vol. 20 , p. 660 .
Verderen. -- Mécanique des sols ef fondations. Eypolles, édit.

WaLz (K.). - Determination of the particle shape of aggregates Betonstrasse 1936. 11 (2), 27-32.

\section{HYDRAULIQUE.}

La bibliographie à consulter est abondante et nous citerons seulement :

Bagnold (R. A.). - The physies of Blown sand on desert 1)unes. London.

Blancher, - Formation et destruction par un courant d'eau de massif's en pierres. La Houlle Blanche, n"2, mars 1946 .

Buprack (W.). - Contribution a la théoric du lavage. Revue de l'Industrie minérale, 1936, pp. 740-750.

Ghatr (Mr.). - Matiere en suspension dans les eaux des fleunes et spheres dans les liquides. Paris, GanthierVillars, 1935.

Hrusstron (F.). - Bulletin Geol. Inst. of Upsala, 25 (1936).

IsBAch. - Les barrages de pierres lancées dans l'eau courante. Extrait des Annales de l'Institut Hydrotech. de recherches scientifiques en U.R.S.S., T. XVII, 1935, p. 12.

lirumbers. - Fundamental attributes of sedimentary particles. Proce. of second Hydraulic Conference, 1942, Unirersité de Iowa.

Me Nown and J. Maralka. - Effects of particle shape on settling velocity at low Reynolds numbers. Transaction of the American Geophysical Union, Vol, 31, pp. 74-82, 1950 .

Rouse. - Monogramme pour la détermination de la vitesse de sédimentation des sphères. Soil Conservation Service, oct. 1937.

Waber.. - The coefficient of resistance as a function of Reynolds number for solid of various shapes. Journal Franklin Institute, Vol. 217 (1934), pp. 459-490.

\section{I S U S I O N}

M. Ne Nown souligne l'intérêt du travail présenté par M. Durand et insiste sul les deux éléments de base qui doivent intervenir dans le choix des différents facteurs cle forme pour chaque etude particulière: la simplicité it la fois de la deffnition et des mesures, et la variation nette et systématique des earactéristiques avec les phénomènes etudiés; par exemple, un chercheur qui est intéressé par la vitesse de chute ne peut employer le mèmo facteur pour des particules suivant des lois de chute très différentes.

En particulier, une particule en forme d'aiguille présentera sa résistance minima à l'avancement dans la direction de son grand axe; dans toute autre direction la resistance sera plus grande et pourtant la forme reste identique. Mar. Malarka et Mc Nown ont été amenés de ce fait a la notion d'olientation et ont trouve que la forme elle-mène pouvait se caracteriser par des modules analogues à ceux employés par M. Duravo. Ils ont en outre constaté que pour des nombres de Reynolds inférieurs a 0,1 toutes les positions etaient stables pour lo mouvement en chute libre; ensuite jusqu'à 300 , la seule position stable est celle correspondant au maître couple maximum; au-dessus de 300 l'instabilité est systématique et il n'est plus possible de définir une position 
moyenne sinon statistiquement. Les considérations d'orientation perdraient beaucoup de leur importance pour l'étude des bétons, et il est évident que l'on ne pent trouver de facteurs de forme qui puissent satisfaire a la fois géologues, ingénieurs et hydrauliciens.

M. Robert Dunawd est d'accord avec Me Nown sur l'influence de l'orientation, que l'on peut souvent constater dans les mesures.

M. Garier remeleie vivement et mapplle que II. Me Nown, Professeur a l'Université d'lowa est en mission d'etudes a Grenoble, tandis que M. Craya accomplit une mission symétrique à l'Université d'Towa. M. Me Nown est un des hydranliciens américains les plus éminents, particulièrement dans le domaine qui nous occupe. Le Comité technique est extrêmement heureux d'accueillir ses observations autorisées et qui représentent bien le point de vue des hydrauliciens dans ce domaine.

M. Cambevx nous a adressé, après la séance, les obserrations suivantes:

«Il ne faudrait pas croire que la sphère soit la forme vers laquelle tendent, par usure, tous les objets détritiques. Seuls, tendent vers la sphère, les galets soumis à une longue erosion tourbillonnaire (galets de marmite, perles de caverne, etc.) et les grains de 0,4 a $5 \mathrm{~mm}$. oolithiques, formés d'écalles concentriques (sables des plages marines au voisinage d'Alexandrie et des plages du Grand Lac salé, U.S.A.). Sur les plages marines à forte houles, les galets tendent, non ver's la sphère, mais vers des ellipsoides aplatis; l'aplatissement augmente quand on passe des quartz aux calcaires ot de la aux schistes. Dans les fleuves, le galet tend vers wne forme limite, d'autant plus usée que le fleuve est plus violent dans ses crues, et qui, ellipsoïlale pour de très rares toments comme la Saoura, est d'ailleurs ovoüde ou simplement polyédrique émoussée. Sous l'action de la seule usure glaciaire, les objets tendent vers le polyèdre émoussé; dans les éboulis et coulées boueuses, l'émoussé est a peine sensible, et la forme demeure polyédrique, ou du moins conforme à l'allure originelle. J'ai donné, en diverses publications, des valeurs numériques d'émoussé, aplatissement et dissymétric, relatifs à ees différents cas.

« Pour apprécier l'allure d'un galet, l'assimilation à une sphère, proposée par certains auteurs, n'a aucune signification réelle pour le géologue, sauf dans Je cas des marmites torrentielles, somme toute fort exceptionnel; la comparaison avec l'ellipsoïde à trois axes inégaux semble meilleure; mais la comparaison avec la figure géométrique originelle (souvent proche d'un parallépipede) serait non moins intéressante.

"Détail de présentation : les notations $L^{\prime}$ ef $L^{\prime}$ " sont l'occasion de frequentes coquilles typographiques et confusions, et on ne devait y recourir quapres avoir épuisé foutes les lettres de l'alphabet.

\& M. Calllevx et M. Thicart félicitent M. Dumand de son travail, qui non seulement foumit une mise au point critique d'une grande clarté, mais encore apporte en plusieurs domaines des idées nouvelles très intéressantes. 》

M. le Président conclut que la variété des coefficients qu'à indiqués M. R. Dunand montre qu'il y a là un vaste champ à défricher, et le remercie pour toute la documentation qu'il a apportée à ce sujet.



